\title{
Intestinal epithelial cells promote secretion of leptin and adiponectin in adipocytes
}

Rino Ishihara ${ }^{\mathrm{a}}$, Yuki Mizuno ${ }^{\mathrm{a}}$, Akiho Miwa ${ }^{\mathrm{a}}$, Akihiro Hamada ${ }^{\mathrm{a}}$, Takeshi Tsuruta ${ }^{\mathrm{b}}$, Martin

Wabitsch $^{\mathrm{c}}$ and Kei Sonoyama ${ }^{\mathrm{b}, *}$

${ }^{\mathrm{a}}$ Graduate School of Life Science, and ${ }^{\mathrm{b}}$ Research Faculty of Agriculture, Hokkaido University, Sapporo 060-8589, Japan

${ }^{\mathrm{c}}$ Division of Pediatric Endocrinology and Diabetes, Department of Pediatrics and Adolescent Medicine, University of Ulm, Ulm 89075, Germany

No. of Tables: 4,575

No. of Figures: 4

No. of Supplementary Tables: 1

No. of Supplementary Figures: 3

*Corresponding author: Kei Sonoyama

Laboratory of Food Biochemistry, Research Faculty of Agriculture, Hokkaido University Kita-9 Nishi-9, Kita-ku, Sapporo 060-8589, Japan

Tel \& Fax: +81-11-706-2496; E-mail: ksnym@chem.agr.hokudai.ac.jp 


\begin{abstract}
Although leptin and adiponectin are the predominant adipokines, how their circulating levels are regulated is incompletely understood. The present study tested whether intestinal epithelial cells influence the expression and secretion of these adipokines by adipocytes. Leptin gene expression and secretion by cultured human primary adipocytes and Simpson-Golabi-Behmel Syndrome adipocytes increased upon coculture with human enterocytic Caco-2 cells or incubation in conditioned medium of Caco-2 cells. Although adiponectin secretion increased, its mRNA levels decreased. Tissue homogenate of the ileum (but not the jejunum, colon, or liver) of nonobese C57BL/6J mice also stimulated leptin and adiponectin secretion by cultured murine 3T3-L1 adipocytes. However, ileal homogenate of obese KK-Ay mice had no effect on leptin and adiponectin secretion. We propose that as yet unidentified humoral factors released from intestinal epithelial cells are involved in regulating circulating leptin and adiponectin levels. Decreased production of such factors may contribute to hyperphagia in KK-Ay mice.
\end{abstract}

Keywords: Leptin; Adiponectin; Adipocyte; Intestine 


\section{Introduction}

White adipose tissue is an endocrine organ that secretes a wide range of protein signals known as adipokines. Leptin, which functions primarily in inhibiting the development of obesity, is a prototypic adipokine that acts through receptors on specific populations of neurons in the brain [1]. The most important factors influencing leptin expression and secretion are the distribution of fat depots and the status of its energy stores because leptin is predominantly expressed in adipocytes, and indeed, circulating leptin concentrations in the fed state are highly correlated with the degree of adiposity [2-4]. In addition, leptin expression and secretion are reportedly promoted by insulin [5-9], glucocorticoids [9, 10] and glucose and/or its metabolites $[11,12]$. Furthermore, infections and inflammation are thought to promote leptin expression and secretion [13-17]. Adiponectin is another major adipokine that is exclusively expressed in adipocytes; in contrast with leptin, circulating adiponectin levels are decreased in obesity [18]. Although much is known about leptin and adiponectin, how their circulating levels are regulated is incompletely understood.

Barbier et al. [19] reported that plasma leptin concentrations increase in rats with trinitrobenzene sulfonic acid-induced colitis or indomethacin-induced ulcerative ileitis, suggesting that mediators released from inflamed intestinal tissue promote leptin expression and secretion by adipocytes. Indeed, leptin expression and secretion are reportedly stimulated by intestinal inflammation-associated inflammatory cytokines such as interleukin (IL)-1 $\alpha$, IL-1 $\beta$, IL-6 and tumor necrosis factor (TNF)- $\alpha$ [13-17]. However, it is unclear whether leptin expression and secretion are regulated by the intestine under physiologic conditions. Previous studies demonstrated that circulating leptin levels decline after fasting and increase after refeeding [20-23]. The evidence led us to conclude that normal intestinal tissue also influences leptin expression and secretion by adipocytes, although it is conceivable that diet-derived substances such as glucose could stimulate leptin expression and secretion by these cells. Thus, we hypothesized that intestinal epithelial cells regulate adipokine expression and secretion by adipocytes via various as yet unidentified humoral factors. The present study tested this 
hypothesis using cell culture experiments.

\section{Materials and methods}

\subsection{Cell culture}

Caco-2 human colon carcinoma cells and 3T3-L1 murine preadipocytes were obtained from the American Type Culture Collection. Simpson-Golabi-Behmel Syndrome (SGBS) human preadipocytes were provided by the Division of Pediatric Endocrinology and Diabetes, Department of Pediatrics and Adolescent Medicine, University of Ulm [24]. Caco-2 cells were maintained as previously described [25]. To induce enterocytic differentiation, subconfluent Caco-2 cells were plated onto filter inserts (BD Falcon) in 6-well plastic plates at an initial density of $0.5 \times 10^{6}$ cells/well. Upon reaching confluence (day 0 ), the cells were cultured for up to 21 days. The transepithelial electrical resistance (TEER) of the Caco-2 monolayer was measured as a marker of enterocytic differentiation [26] using a Millicell-ERS (Merck Millipore). Culture and differentiation of 3T3-L1 and SGBS preadipocytes into adipocytes were performed according to Frost \& Lane [27] and Fischer-Posovszky et al. [28], respectively. Culture and differentiation of primary human visceral preadipocytes (poietics human visceral preadipocytes, Lonza) into adipocytes were performed according to the supplier's instructions. 3T3-L1 cells, SGBS cells and primary human visceral preadipocytes were used as differentiated adipocytes 12, 20, and 10 days after induction of differentiation, respectively. Adipocytic differentiation was confirmed by monitoring cells for lipid droplet formation using phase-contrast microscopy (CKX41N-31PHP, Olympus, data not shown).

\subsection{Animals and sampling}

All study protocols were approved by the Animal Use Committee of Hokkaido University (approval no. 08-0139). Animals were maintained in accordance with the Hokkaido University guidelines for the care and use of laboratory animals. Male C57BL/6J JmsSlc (B6, age 5 weeks) and KK-Ay/TaJcl (KK, age 10 weeks) mice were purchased from Japan SLC and CLEA Japan, respectively. Mice were housed in standard plastic cages in a temperature-controlled $\left(23 \pm 2{ }^{\circ} \mathrm{C}\right)$ 
room under a 12-h light/dark cycle and were allowed free access to water and standard chow $\operatorname{diet}(\mathrm{MR}$ stock, Nosan Corporation) for 2 weeks. On the last day of feeding, mice were euthanized by exsanguination via severing of the carotid artery under sevoflurane anesthesia. A laparotomy was made and the liver, small intestine, and colon were excised. The proximal and distal halves of the small intestine were defined as the jejunum and ileum, respectively. The luminal contents were thoroughly washed out with ice-cold saline, the jejunum, ileum, and colon were opened longitudinally, and the mucosa was scraped off using a glass slide. Samples of liver and intestinal mucosa were homogenized in ice-cold serum-free DMEM supplemented with penicillin $\mathrm{G}$ potassium $(100 \mathrm{U} / \mathrm{mL})$, streptomycin sulfate $(100 \mu \mathrm{g} / \mathrm{mL})$ and gentamicin sulfate $(50 \mu \mathrm{g} / \mathrm{mL})$. The homogenates were centrifuged at $14,000 \times g$ at $4^{\circ} \mathrm{C}$ for $5 \mathrm{~min}$, and the supernatants were filtered using a $0.2-\mu \mathrm{m}$ syringe filter (Acrodisc). The protein concentration of each sample was measured using a BCA protein assay kit (Thermo Scientific) according to the manufacturer's instructions. All samples were then stored at $-80^{\circ} \mathrm{C}$ until use.

\subsection{Experimental design}

For coculture of adipocytes with intestinal epithelial cells, filter inserts on which Caco-2 cells were cultured were placed in the wells of 6-well plates in which adipocytes were cultured (Supplementary Fig. 1A). Both Caco-2 cells and adipocytes were cultured in serum-free RPMI 1640 medium during the pre-coculture and coculture periods (10 and $24 \mathrm{~h}$, respectively). After coculture, the medium was removed from the basal compartment of the filter inserts and subjected to adipokine determination as described below. RNA was isolated from the cells as described below. Adipocytes that were not cocultured (i.e., monocultures) were cultured in serum-free medium for the same amount of time as the pre-coculture and coculture periods. To culture adipocytes in conditioned medium (CM) of Caco-2 cells, CM was removed from the basolateral compartment of filter inserts on which Caco-2 cells had been cultured in serum-free RPMI 1640 medium for 24 h (Supplementary Fig. 1B). Adipocytes were cultured with serum-free medium supplemented with different concentrations of CM for $0,15,24$ or $48 \mathrm{~h}$, after which the medium was subjected to adipokine determination as described below. Cells 
were subjected to determination of total triglycerides (TGs) as described below. Unless otherwise noted, Caco-2 cells at least 14 days after reaching confluence were used for coculture and CM experiments. 3T3-L1 adipocytes were also cultured for $24 \mathrm{~h}$ in serum-free DMEM supplemented with the filtrates of murine tissue homogenates prepared as described above (Supplementary Fig. 1C).

\subsection{ELISA}

The concentrations of adiponectin, leptin and resistin in the culture medium were determined using ELISA kits (Adiponectin/Acrp30 DuoSet, Leptin DuoSet ELISA Development and Resistin Quantikine ELISA kit, R\&D Systems) according to the manufacturers' instructions.

\subsection{Colorimetric determination of TGs in adipocytes}

After removal of the medium, SGBS cells were lysed by sonication in a buffer composed of 50 $\mathrm{mM}$ Tris- $\mathrm{HCl}(\mathrm{pH} 7.4), 150 \mathrm{mM} \mathrm{NaCl}, 0.1 \%$ (w/v) sodium dodecyl sulfate, $1 \%(\mathrm{w} / \mathrm{v})$ Triton $\mathrm{X}-100$ and $1 \%(\mathrm{w} / \mathrm{v})$ sodium deoxycholate. The concentration of TGs in the lysates was measured using a Triglyceride E-Test (Wako Pure Chemical Industries) according to the manufacturer's instructions.

\subsection{Analysis of $m R N A$ expression}

Total RNA was isolated from adipocytes using a ReliaPrep RNA Cell Miniprep System (Promega) and reverse transcribed to generate first-strand cDNA using a ReverTra Ace qPCR RT kit (Toyobo) according to the manufacturer's instructions. To compare the steady-state levels of mRNAs encoding adipocytokines and adipocytic differentiation-related proteins, real-time quantitative PCR (RT-qPCR) was performed using GeneAce SYBR qPCR Mix $\alpha$ No ROX (Nippon Gene) with a Thermal Cycler Dice TP800 (Takara Bio) according to the manufacturer's instructions. The sequences of primers used for RT-qPCR are shown in Supplementary Table 1. Relative mRNA expression levels for each sample were normalized to that of glyceraldehyde 3-phosphate dehydrogenase (GAPDH). 


\subsection{Statistical analysis}

Results are presented as means and SEM. Unpaired $t$-tests were used to compare means between two groups. Dunnet's or Tukey-Kramer's multiple comparison tests following one-way analysis of variance was used to compare mean values between three or more groups. Data were analyzed using GraphPad Prism for Macintosh (version 6, GraphPad Software). $P$ values $<0.05$ were considered to indicate statistical significance.

\section{Results}

\subsection{Caco-2 cells promote leptin and adiponectin secretion by adipocytes}

The concentration of leptin was higher in the culture supernatant of human primary visceral adipocytes cocultured with Caco- 2 cells without direct contact for $24 \mathrm{~h}$ than in the culture supernatant of monocultured adipocytes (Fig. 1A). In addition, culture of primary adipocytes with CM of Caco- 2 cells for $24 \mathrm{~h}$ increased the concentration of leptin in the culture supernatant (Fig. 1B). Likewise, the leptin concentration was significantly higher in the culture supernatant of SGBS adipocytes cocultured with Caco-2 cells than in the supernatant of monocultured SGBS adipocytes (Fig. 1C). The leptin concentration in the supernatant of SGBS adipocytes cultured in CM of Caco-2 cells increased in both a time- and concentration-dependent manner (Fig. 1D). No leptin was detected in CM of Caco-2 cells (data not shown).

The adiponectin concentration also increased in the culture supernatant of SGBS adipocytes following coculture with Caco-2 cells or culture in CM of Caco-2 cells (Fig. 1E and 1F), whereas no resistin was detectable in the culture supernatant (data not shown). Boiling CM for 10 min had no effect on the leptin concentration, which was still significantly higher in the culture supernatant of SGBS adipocytes cultured in boiled CM than in the supernatant of cells cultured without CM, and the concentration was similar to that in the supernatant of cells cultured in unheated CM (Supplementary Fig. 2). The TG concentration in SGBS adipocytes cultured in various concentrations of CM for 24 and 48 h was the same (Supplementary Fig. 
3).

\subsection{Caco-2 cells promote expression of the leptin gene in SGBS adipocytes}

Similar to the increased concentration of leptin observed in the culture supernatant, leptin mRNA levels as estimated by RT-qPCR were significantly higher in SGBS adipocytes cocultured with Caco-2 cells for $24 \mathrm{~h}$ than in monocultured SGBS adipocytes (Fig. 2). In contrast, adiponectin mRNA levels in SGBS adipocytes declined significantly following coculture with Caco-2 cells. With respect to mRNA levels for adipocytic differentiation-related genes (i.e., peroxisome proliferator-activated receptor $\gamma$ [PPAR $\gamma]$, CCAAT enhancer binding protein $\alpha[\mathrm{C} / \mathrm{EBP} \alpha]$, acetyl CoA carboxylase [ACC], fatty acid synthase [FAS] and lipoprotein lipase [LPL]), there were no significant differences between cocultured and monocultured SGBS adipocytes, although the levels were consistently lower in cocultured compared with monocultured cells.

\subsection{Differentiation of Caco-2 cells influences the stimulatory effect on leptin and adiponectin} secretion by $S G B S$ adipocytes

TEER is known to increase with enterocytic differentiation of Caco-2 cells [26]. In the present study, the TEER continued to increase after the cells reached confluence, plateauing on day 17 (Fig. 3A). Leptin and adiponectin concentrations were significantly higher in the culture supernatant of SGBS adipocytes cultured in CM of 21-day post-confluent Caco-2 cells than in the supernatant of cells cultured without CM supplementation (Fig. 3B and 3C). There were no significant differences in the leptin and adiponectin concentrations between SGBS adipocytes cultured in CM of 1-day post-confluent Caco-2 cells and those cultured without CM supplementation.

\subsection{Murine ileum stimulates the secretion of leptin and adiponectin by murine adipocytes} Tissue homogenates were prepared from the jejunum, ileum, colon and liver of nonobese B6 and obese KK mice. The mean body weight of the B6 and KK mice was approximately 22 and 
$40 \mathrm{~g}$, respectively. 3T3-L1 murine adipocytes were cultured for $24 \mathrm{~h}$ in serum-free medium supplemented with filtrates of tissue homogenates adjusted to a protein concentration of 50 $\mu \mathrm{g} / \mathrm{mL}$. The concentration of leptin (and to a lesser extent adiponectin) in the culture supernatant was significantly higher in 3T3-L1 adipocytes cultured with ileal mucosa homogenate of B6 mice than in those cultured without homogenate (Fig. 4A and 4B). There were no differences in the leptin and adiponectin concentrations in the supernatant of 3T3-L1 adipocytes cultured with jejunal, colonic and hepatic homogenates of B6 mice compared with cells cultured without homogenate. In KK mice, there were no significant differences in the culture supernatant leptin and adiponectin concentrations between 3T3-L1 adipocytes cultured with jejunal, ileal or colonic homogenate and cells cultured without homogenate (Fig. 4C and 4D). The concentration of leptin (and to a lesser extent adiponectin) was significantly higher in the supernatant of 3T3-L1 adipocytes cultured with B6 ileal homogenate than in the supernatant of cells cultured with KK ileal homogenate.

\section{Discussion}

The present study clearly demonstrated that leptin expression and secretion by adipocytes is upregulated by coculture with Caco-2 intestinal epithelial cells at the pretranslational stage. Because Caco-2 cell CM also promoted the upregulation of leptin secretion by adipocytes, it appears likely that as yet unidentified humoral factors produced by Caco- 2 cells promote leptin expression and secretion by adipocytes.

The present study employed adipocytes differentiated from human primary preadipocytes, SGBS preadipocytes, and 3T3-L1 preadipocytes. Therefore, one may suspect that coculture with Caco-2 cells and CM of Caco-2 cells merely promote adipocytic differentiation in preadipocytes. However, coculture with Caco-2 cells led to an increase in the level of leptin mRNA in SGBS cells, but not to increases in the levels of mRNAs of adipocytic differentiation-related genes such as PPAR $\gamma, \mathrm{C} / \mathrm{EBP} \alpha, \mathrm{ACC}, \mathrm{FAS}$ and LPL. In addition, Caco-2 cell CM had no effect on the concentration of TGs in SGBS cells. Therefore, it appears unlikely 
that the observed Caco-2 cell-induced increases in leptin expression and secretion reflect accelerated differentiation of adipocytes.

In the present study, tissue homogenate prepared from the ileal mucosa of B6 mice stimulated leptin secretion by murine 3T3-L1 adipocytes. This observation also suggests that one or more substances derived from the intestine promote leptin secretion by adipocytes. It is noteworthy that leptin secretion was stimulated by ileal tissue homogenate but not by jejunal or colonic homogenate. This observation led us to conclude that the link between intestinal epithelial cells and leptin secretion by adipocytes involves the so-called "ileal brake." Under normal physiologic conditions, the ileal brake is induced when undigested nutrients reach the ileum, leading to reduced food intake and increased satiety [29]. Peptide YY (PYY) and glucagon-like peptide-1 (GLP-1), which are peptide hormones secreted by enteroendocrine L cells in the ileum and colon, are the most likely putative mediators of the ileal brake [29]. The effect of PYY and GLP-1 on food intake is thought to be paracrine in nature, affecting local vagal afferent nerves and in turn the central nervous system, rather than the endocrine system $[29,30]$. From the present observations, however, one could conclude that PYY and/or GLP-1 released from the ileum induce the ileal brake by promoting the secretion of leptin (a satiety hormone) by adipocytes. However, no evidence indicating that Caco-2 cells produce PYY and/or GLP-1, or that adipocytes express receptors for PYY and/or GLP-1, has been reported. Because levels of circulating leptin decrease after fasting and increases after refeeding [20-23], our findings suggest that ileum-derived humoral factors other than PYY and GLP-1 are released in response to nutrients reaching the ileum to induce leptin secretion by adipocytes. Such humoral factors could be considered novel putative mediators of the ileal brake.

Although ileal homogenate of nonobese B6 mice stimulated leptin secretion by 3T3-L1 adipocytes, ileal homogenate of obese KK mice did not. KK mice are used as a polygenic model for type 2 diabetes and show obesity due to hyperphagia [31]. Given that the ileum-derived humoral factors stimulate leptin secretion, which in turn increases the level of 
satiety, reduced production of the ileum-derived humoral factors may contribute to hyperphagia in KK mice. In other words, hyperphagia in $\mathrm{KK}$ mice may be due to failure of the ileal brake induced by the ileum-derived humoral factors.

Coculture with Caco- 2 cells led to an increase in the concentration of adiponectin in the culture supernatant of SGBS adipocytes and a decrease in the level of adiponectin mRNA. In addition, ileal mucosa tissue homogenate of B6 mice stimulated adiponectin secretion by murine 3T3-L1 adipocytes. These observations suggest that adiponectin secretion is also promoted by intestinal epithelial cells. In addition, considering that circulating adiponectin levels decline in obesity [18], the present observation that ileal mucosa tissue homogenate of obese KK mice did not stimulate adiponectin secretion by 3T3-L1 adipocytes suggests that reduced production of the ileum-derived humoral factors contributes to the decrease in circulating levels of adiponectin in obesity.

The ileum-derived humoral factors remain unidentified. In the present study, CM of differentiated (but not undifferentiated) Caco-2 cells stimulated the secretion of leptin and adiponectin by SGBS adipocytes, suggesting that differentiated enterocytes produce the humoral factors that are responsible for inducing the secretion of adipokines. Leptin expression and secretion are reportedly promoted by insulin [5-9], glucocorticoids [9, 10], glucose and/or its metabolites $[11,12]$ and inflammatory cytokines, including IL1- $\alpha$, IL-1 $\beta$, IL-6 and TNF- $\alpha$ [13-17]. Because boiling of Caco-2 cell CM had no effect on leptin secretion-stimulating activity, it seems unlikely that the ileum-derived humoral factors are proteinaceous substances such as insulin or cytokines. In addition, because no difference was observed between Caco-2 cell $\mathrm{CM}$ and SGBS cell culture supernatant with respect to glucose concentration $(8.8 \mathrm{mM} v \mathrm{~s}$. $11.4 \mathrm{mM}$, respectively), glucose can also be eliminated as a candidate. Sidler et al. [32] reported that colon cancer cell lines (including Caco-2 cells) produce cortisol. Therefore, it would be interesting to test whether ileal epithelial cells secrete glucocorticoids in response to nutrients, in turn stimulating leptin production in adipocytes. 
In conclusion, our findings suggest that as yet unidentified humoral factors released from differentiated enterocytes in the ileum are involved in regulating the expression and secretion of leptin and adiponectin by adipocytes. Our findings in KK mice also suggest that reduced production of such factors may contribute to hyperphagia. Further studies are necessary to identify and characterize these factors.

\section{Acknowledgments}

This study was supported in part by a Grant-in-Aid for Scientific Research from the Ministry of Education, Science, Sports and Culture of Japan (no. 26292062), by the Regional Innovation Strategy Support Program of the MEXT, and by the Center of Innovation Program Trial, Japan Science and Technology Agency. The authors have no conflict of interest to disclose. 


\section{References}

1. H. Pan, J. Guo, Z. Su, Advances in understanding the interrelations between leptin resistance and obesity. Physiol. Behav. 130 (2014) 157-169.

2. R.C. Frederich, A. Hamann, S. Anderson, et al., Leptin levels reflect body lipid content in mice: evidence for diet-induced resistance to leptin action. Nat. Med. 1(1995) 1311-1314.

3. B.S. Hamilton, D. Paglia, A.Y.M. Kwan, et al., Increased obese mRNA expression in omental fat cells from massively obese humans. Nat. Med. 1 (1995) 953-956.

4. M. Maffei, J. Halaas, E. Ravussin, et al., Leptin levels in human and rodent: measurement of plasma leptin and $o b$ RNA in obese and weight-reduced subjects. Nat. Med. 1 (1995) $1155-1161$.

5. H. Fukuda, N. Iritani, Regulation of ATP citrate lyase gene expression in hepatocytes and adipocytes in normal and genetically obese rats. J. Biochem. 126 (1999) 437-444.

6. S.J. Koopmans, M. Frolich, E.H. Gribnau, et al., Effect of hyperinsulinemia on plasma leptin concentrations and food intake in rats. Am. J. Physiol. Endocrinol. Metab. 274 (1998) E998-E1001.

7. O.A. MacDougald, C.S. Hwang, H.Y. Fan, et al., Regulated expression of the obese gene product (leptin) in white adipose tissue and 3T3-L1 adipocytes. Proc. Natl. Acad. Sci. U. S. A. 92 (1995) 9034-9037.

8. R. Saladin, P.D. Vos, G.M. Michele, et al., Transient increase in obese gene expression after food intake or insulin administration. Nature 377 (1995) 527-529.

9. M. Wabitsch, P.B. Jensen, W.F. Blum, et al., Insulin and cortisol promote leptin production in cultured human fat cells. Diabetes 45(1996) 1435-1438.

10. T. Murakami, M. Iida, K. Shima, Dexamethasone regulates obese expression in isolated rat adipocytes. Biochem. Biophys. Res. Commun. 214 (1995) 126-127.

11. W.M. Mueller, F.M. Gregoire, K.L. Stanhope, et al., Evidence that glucose metabolism regulates leptin secretion from cultured rat adipocytes. Endocrinology 139 (1998) 551-558.

12. P. Wellhoener, B. Fruehwald-Schultes, W. Kern, et al., Glucose metabolism rather than insulin is a main determinant of leptin secretion in humans. J. Clin. Endocrinol. Metab. 85 
(2000) 1267-1271.

13. J.M. Bruun, S.B. Pedersen, K. Kristensen, et al., Effects of pro-inflammatory cytokines and chemokines on leptin production in human adipose tissue in vitro. Mol. Cell. Endocrinol. 190 (2002) 91-99.

14. C.C. Gerhardt, I.A. Romero, R. Cancello, et al., Chemokines control fat accumulation and leptin secretion by cultured human adipocytes. Mol. Cell. Endocrinol. 175 (2001) 81-92.

15. C. Grunfeld, C. Zhao, J. Fuller, et al., Endotoxin and cytokines induce expression of leptin, the $o b$ gene product, in hamsters. J. Clin. Invest. 97 (1996) 2152-2157.

16. P. Sarraf, R.C. Frederich, E.M. Turner, et al., Multiple cytokines and acute inflammation raise mouse leptin levels: potential role in inflammatory anorexia. J. Exp. Med. 185 (1997) 171-175.

17. P.J. Simons, P.S. van den Pangaart, C.P.A.A. van Roomen, et al., Cytokine-mediated modulation of leptin and adiponectin secretion during in vitro adipogenesis: Evidence that tumor necrosis factor- $\alpha$ - and interleukin-1 $\beta$-treated human preadipocytes are potent leptin producers. Cytokine 32 (2005) 94-103.

18. T. Yamauchi, T. Kadowaki, Adiponectin receptor as a key player in healthy longevity and obesity-related diseases. Cell Metab. 17 (2013) 185-196.

19. M. Barbier, C. Cherbut, A.C. Aubé, et al., Elevated plasma leptin concentrations in early stages of experimental intestinal inflammation in rats. Gut 43 (1998) 783-790.

20. B. Ahren, S. Mansson, R.L. Gingerich, et al., Regulation of plasma leptin in mice: influence of age, high-fat diet and fasting. Am. J. Physiol. Regul. Integr. Comp. Physiol. 273 (1997) R113-R120.

21. G. Boden, X. Chen, M. Mozzoli, et al., Effect of fasting on serum leptin in normal human subjects. J. Clin. Endocrinol. Metab. 81 (1996) 3419-3423.

22. L.J. Hardie, D.V. Rayner, S. Holmes, et al., Circulating leptin levels are modulated by fasting, cold exposure and insulin administration in lean but not Zucker $(f a / f a)$ rats as measured by ELISA. Biochem. Biophys. Res. Commun. 223 (1996) 660-665.

23. D.S. Weigle, P.B. Duell, W.E. Connor, et al., Effect of fasting, refeeding, and dietary fat 
restriction on plasma leptin levels. J. Clin. Endocrinol. Metab. 82 (1997) 561-565.

24. M. Wabitsch, R.E. Brenner, I. Melzner, et al., Characterization of a human preadipocyte cell strain with high capacity for adipose differentiation. Int. J. Obes. 25 (2001) 8-15.

25. K. Sonoyama, S. Rutatip, T. Kasai, Gene expression of activin, activin receptors, and follistatin in intestinal epithelial cells. Am. J. Physiol. Gastrointest. Liver Physiol. 278 (2000) G89-G97.

26. F. Delie, W. Rubas, A human colonic cell line sharing similarities with enterocytes as a model to examine oral absorption: advantages and limitations of the Caco-2 model. Crit. Rev. Ther. Drug Carrier Syst. 14 (1997) 221-286.

27. S.C. Frost, M.D. Lane, Evidence for the involvement of vicinal sulfhydryl groups in insulin-activated hexose transport by 3T3-L1 adipocytes. J. Biol. Chem. 260 (1985) $2646-2652$.

28. P. Fischer-Posovszky, H. Hebestreit, A.K. Hofmann, et al., Role of CD95-mediated adipocyte loss in autoimmune lipodystrophy. J. Clin. Endocrinol. Metab. 91 (2006) 1129-1135.

29. P.W.J. Maljaars, H.P.F. Peters, D.J. Mela, et al., Ileal brake: A sensible food target for appetite control. A review. Physiol. Behav. 95 (2008) 271-281.

30. D.J. Drucker, The biology of incretin hormones. Cell Metab. 3 (2006) 153-165.

31. H. Iwatsuka, A. Shino, Z. Suzouki Z, General survey of diabetic features of yellow KK mice. Endocrinol. Japon. 17 (1970) 23-35.

32. D. Sidler, P. Renzulli, C. Schnoz, et al., Colon cancer cells produce immunoregulatory glucocorticoids. Oncogene. 30 (2011) 2411-2419. 


\section{Figure legends}

Figure 1. Effect of coculture with Caco-2 cells on the secretion of leptin and adiponectin by adipocytes. Charts $A-D$ show the leptin concentration in the culture supernatant of human primary adipocytes $(A$ and $B)$ and Simpson-Golabi-Behmel Syndrome (SGBS) adipocytes ( $C$ and $D$ ). Charts $E$ and $F$ show the adiponectin concentration in the culture supernatant of SGBS cells. Human primary adipocytes and SGBS adipocytes were cocultured with $(\mathrm{Caco}-2[+])$ or without (Caco-2 [-]) Caco-2 cells across the filter insert for $24 \mathrm{~h}(A, C$ and $E)$ or cultured in medium supplemented with various concentrations of conditioned medium of Caco-2 cells for $24 \mathrm{~h}(B, D$ and $F)$. The data in charts $B-F$ are expressed as the means and SEM of three independent experiments, whereas the data in chart $A$ represent a single experiment. In charts $C$ and $E$, mean values with an asterisk were significantly different $(p<0.05) v s$. Caco-2 $(-)$ as estimated by unpaired $t$-test. In charts $B$ and $F$, mean values with an asterisk were significantly different $(p<0.05)$ vs. $0 \%$ as estimated by Dunnet's test following one-way analysis of variance (ANOVA). In chart $D$, mean values with an asterisk were significantly different $(p<0.05) v s .0 \mathrm{~h}$ at each concentration of conditioned medium supplement as estimated by Dunnet's test following one-way ANOVA, and mean values with different letters were significantly different $(p<0.05)$ at each time point as estimated by Tukey-Kramer's test following one-way ANOVA.

Figure 2. Effect of coculture with Caco-2 cells on the expression of adipocytokine genes and adipocytic differentiation-related genes in Simpson-Golabi-Behmel Syndrome (SGBS) adipocytes. SGBS adipocytes were cocultured with (Caco-2 $[+])$ or without (Caco-2 $[-])$ Caco-2 cells across the filter insert for $24 \mathrm{~h}$. Data for Caco-2 (+) are shown relative to the levels in Caco-2 (-), which were set to 1. Data are expressed as the means and SEM of three independent experiments. Mean values with an asterisk were significantly different $(p<0.05) v s$. Caco-2 (-) as estimated by unpaired $t$-test. ACC, acetyl CoA carboxylase; C/EBP $\alpha$, CCAAT enhancer binding protein $\alpha$; FAS, fatty acid synthase; LPL, lipoprotein lipase; PPAR $\gamma$, peroxisome proliferator-activated receptor $\gamma$. 
Figure 3. Effect of enterocytic differentiation of Caco-2 cells on the secretion of leptin by adipocytes. Chart $A$ shows the time-course of changes in transepithelial electrical resistance (TEER), a marker of enterocytic differentiation, after Caco-2 cells reached confluence. Charts $B$ and $C$ show the leptin and adiponectin concentrations, respectively, in the culture supernatant of Simpson-Golabi-Behmel Syndrome adipocytes cultured with conditioned medium of Caco-2 cells on days 1 and 21 after reaching confluence. Data are expressed as the means and SEM of three independent experiments. In chart $A$, mean values with different letters were significantly different $(p<0.05)$ as estimated by Tukey-Kramer's test following one-way analysis of variance (ANOVA). In charts $B$ and $C$, mean values with an asterisk were significantly different $(p<0.05)$ vs. cells cultured without conditioned medium (No add) as estimated by Dunnet's test following one-way ANOVA.

Figure 4. Effect of murine intestinal tissue homogenates on the secretion of leptin by murine 3T3-L1 adipocytes. Charts $A$ and $B$ show the leptin and adiponectin concentrations, respectively, in the culture supernatant of 3T3-L1 adipocytes cultured with jejunal, ileal, colonic or liver tissue homogenate of C57BL/6J JmsSlc (B6) mice. Charts $C$ and $D$ show the leptin and adiponectin concentrations, respectively, in the culture supernatant of 3T3-L1 adipocytes cultured with jejunal, ileal, colonic or liver tissue homogenate of B6 and KK-Ay/TaJcl (KK) mice. Data are expressed as the means and SEM of three mice. In charts $A$ and $B$, mean values with an asterisk were significantly different $(p<0.05) v s$. cells cultured without conditioned medium (No add) as estimated by Dunnet's test following one-way analysis of variance. In charts $C$ and $D$, mean values with an asterisk were significantly different $(p<0.05)$ for KK mice $v s$. B6 mice as estimated by unpaired $t$-test. 
A

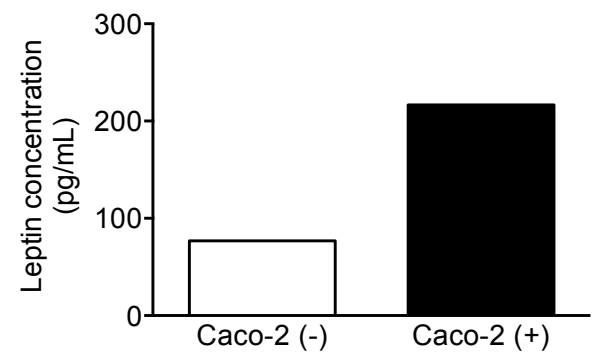

C

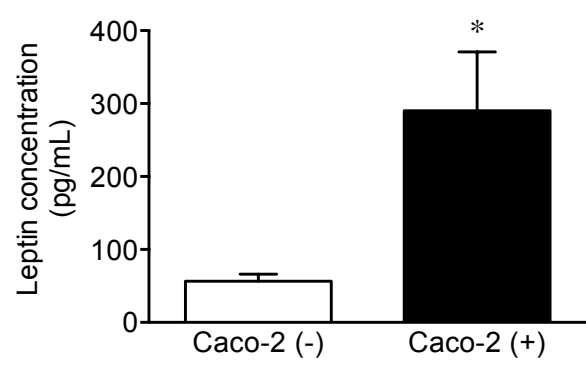

E

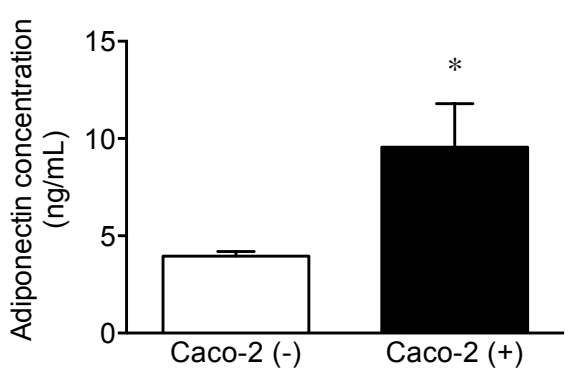

B

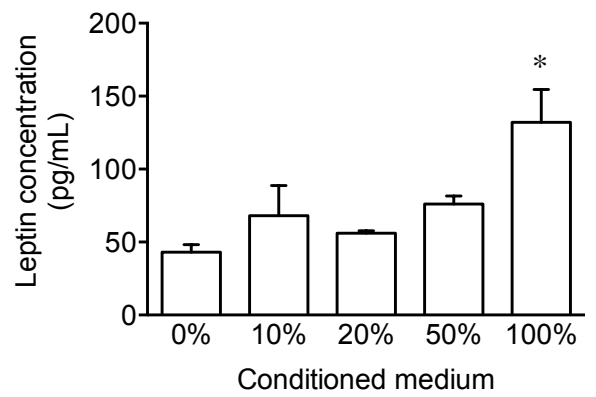

D
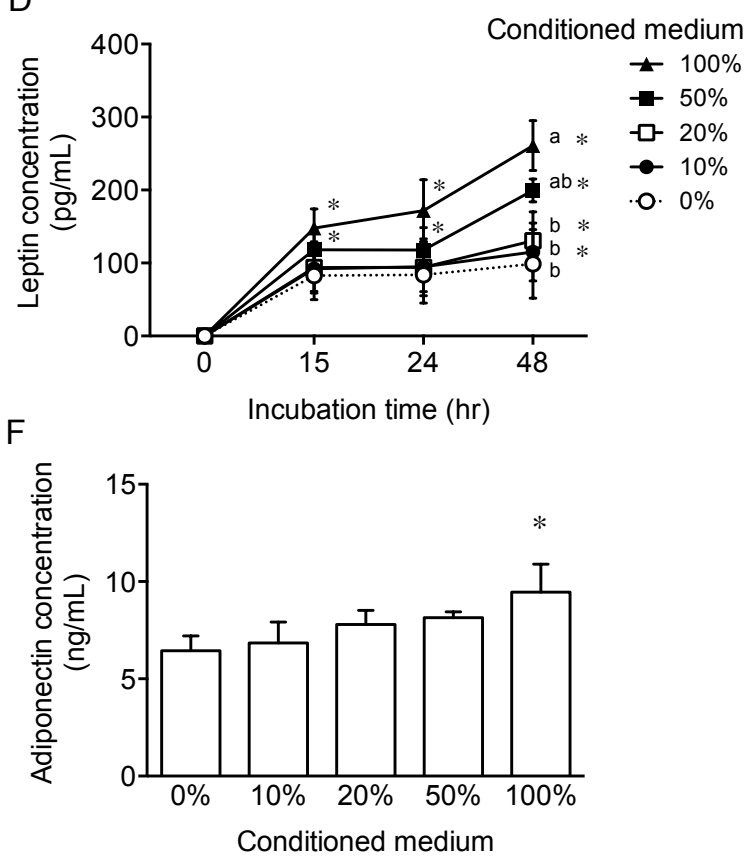

Fig. 1 Ishihara et al.

( 2 column) 

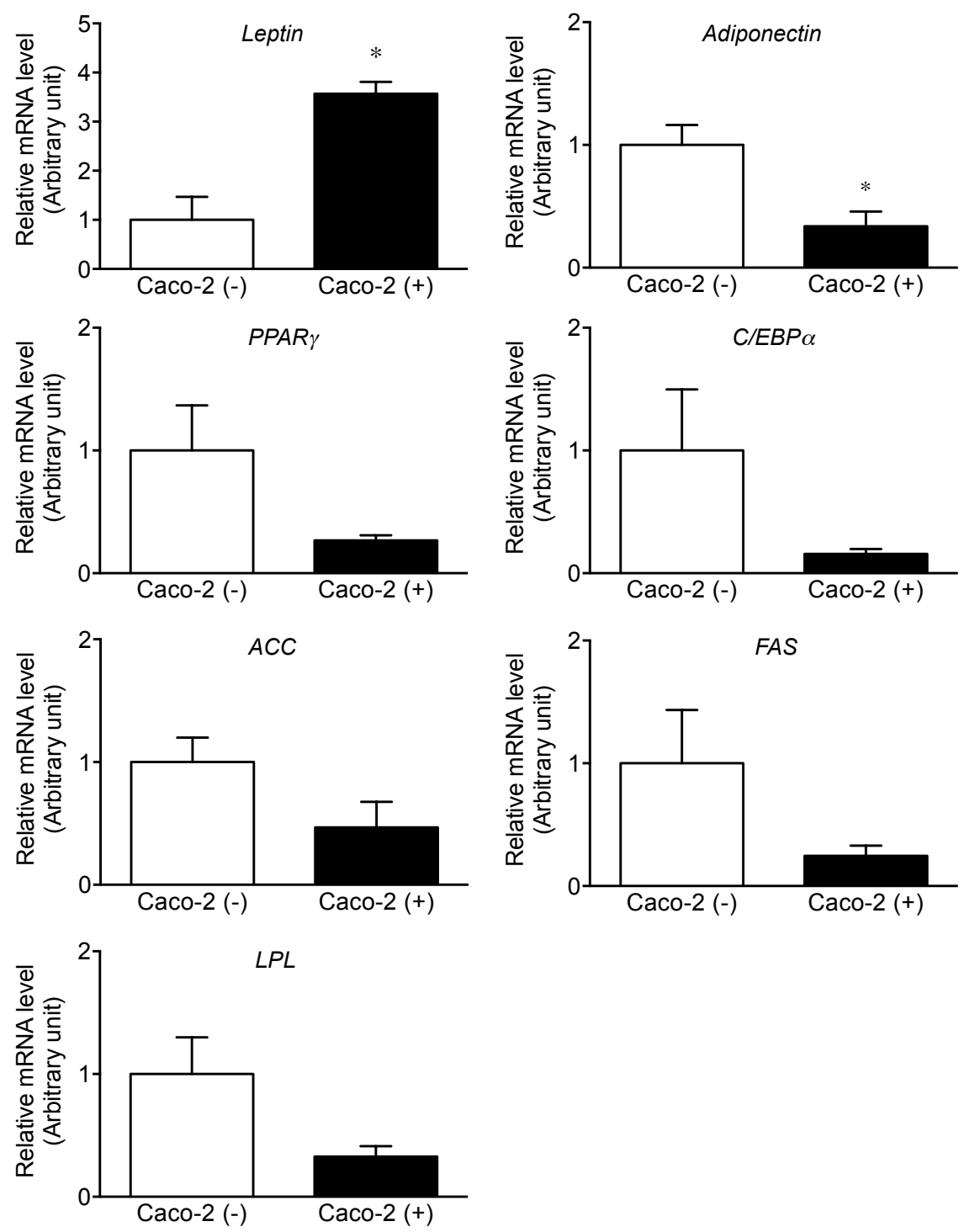

Fig. 2 Ishihara et al.

( 2 column) 


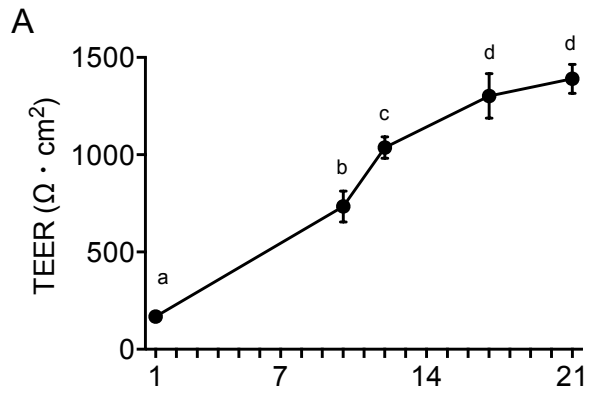

Time after reaching confluence (day)

B

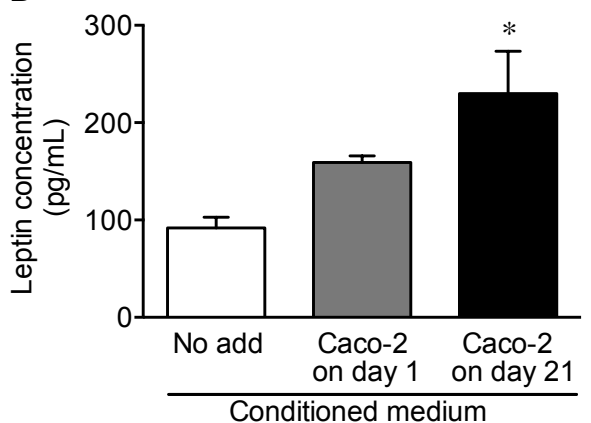

C

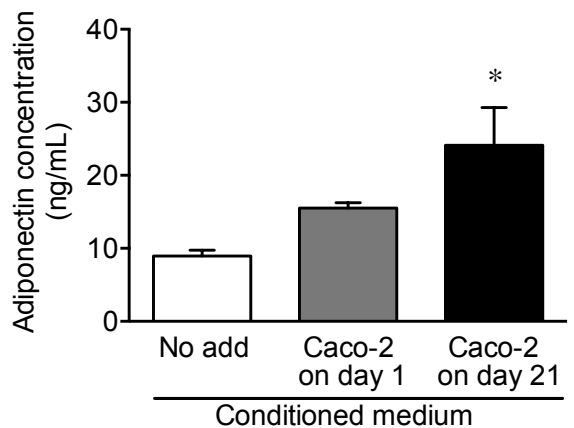

Fig. 3 Ishihara et al.

(single column) 
A

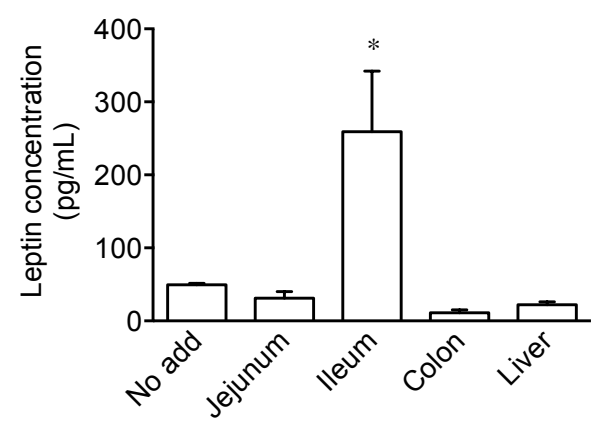

C

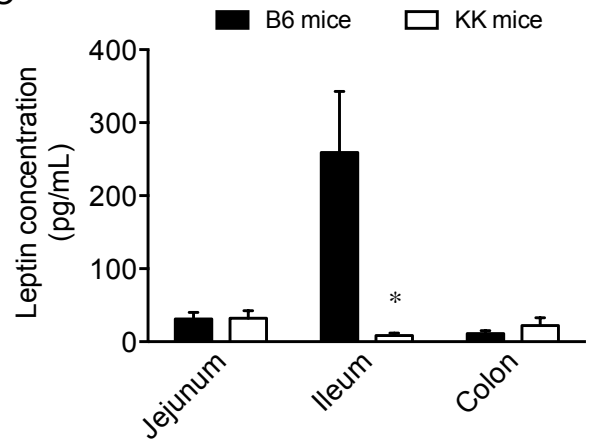

B

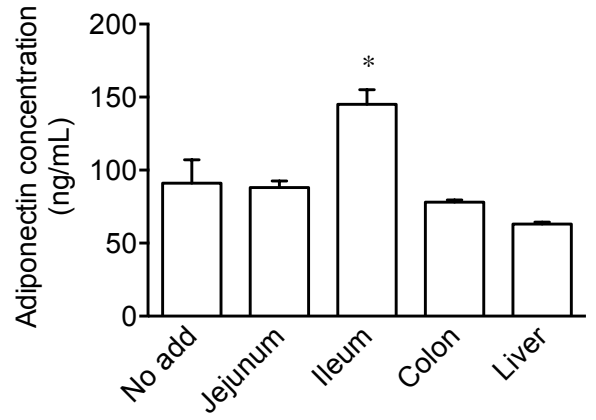

D

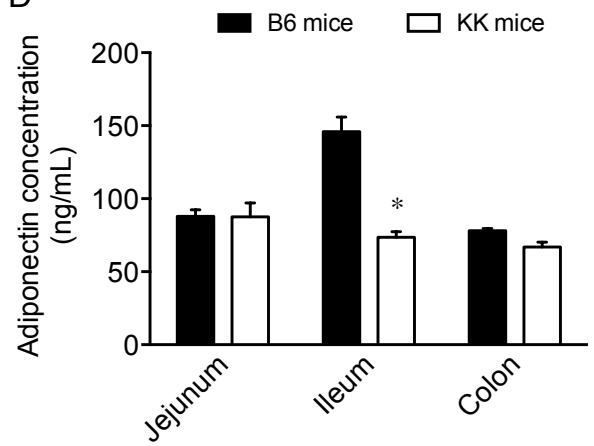

Fig. 4 Ishihara et al.

( 2 column) 